\title{
Electronic Structure of Rare-Earth Metals. II. Positron Annihilation
}

\author{
Williams, R. W.; Mackintosh, Allan
}

Published in:
PHYSICAL REVIEW

Link to article, DOI:

10.1103/PhysRev.168.679

Publication date:

1968

Document Version

Publisher's PDF, also known as Version of record

Link back to DTU Orbit

Citation (APA):

Williams, R. W., \& Mackintosh, A. (1968). Electronic Structure of Rare-Earth Metals. II. Positron Annihilation. PHYSICAL REVIEW, 168(3), 679-686. https://doi.org/10.1103/PhysRev.168.679

\section{General rights}

Copyright and moral rights for the publications made accessible in the public portal are retained by the authors and/or other copyright owners and it is a condition of accessing publications that users recognise and abide by the legal requirements associated with these rights.

- Users may download and print one copy of any publication from the public portal for the purpose of private study or research.

- You may not further distribute the material or use it for any profit-making activity or commercial gain

- You may freely distribute the URL identifying the publication in the public portal

If you believe that this document breaches copyright please contact us providing details, and we will remove access to the work immediately and investigate your claim 


\title{
Electronic Structure of Rare-Earth Metals. II. Positron Annihilation*
}

\author{
R. W. Williams $\dagger$ \\ Institute for Atomic Research and Department of Physics, Iowa State University, Ames, Iowa
}

AND

A. R. Mackintosh

Iowa State University, Ames Iowa, and Technical University, Lyngby, Denmark

(Received 26 October 1967)

\begin{abstract}
The angular correlation of the photons emitted when positrons annihilate with electrons has been studied in single crystals of the rare-earth metals $\mathrm{Y}, \mathrm{Gd}, \mathrm{Tb}, \mathrm{Dy}, \mathrm{Ho}$, and $\mathrm{Er}$, and in a single crystal of an equiatomic alloy of Ho and Er. A comparison of the results for $\mathrm{Y}$ with the calculations of Loucks shows that the independent-particle model gives a good first approximation to the angular distribution, although correlation effects probably smear out some of the structure. The angular distributions from the heavy rareearth metals are very similar to that from $\mathrm{Y}$ and can be understood qualitatively in terms of the relativistic augmented-plane-wave calculations by Keeton and Loucks. The angular distributions in the $c$ direction in the paramagnetic phases are characterized by a rapid drop at low angles followed by a hump, and these features are associated with rather flat regions of Fermi surface normal to the $c$ axis. The same aspects of the Fermi surface are believed to be important in determining the stability of the periodic magnetic structures formed in some of the metals, and there is a strong correlation between the structure in the angular distribution and the magnetic properties. In the spiral phase of $\mathrm{Ho}$, the structure in the $c$-axis distribution is much reduced, indicating that the Fermi surface is substantially modified by the magnetic ordering, as expected. The photon distribution from the equiatomic Ho-Er alloy is very similar to those from the constituent metals, although the structure is somewhat more pronounced.
\end{abstract}

\section{INTRODUCTION}

$T$ HE experiments reported in this paper were undertaken with the aims of critically testing the independent-particle theory of positron annihilation and of elucidating the electronic structure of the heavy rare-earth metals. The theory of the annihilation of positrons with electrons in metals has been extensively discussed recently, especially with regard to the corrections to the simple independent-particle model $^{1}$ which are a consequence of the Coulomb interactions in the electron-positron system..$^{2,3}$ These theories have been concerned primarily with the positron lifetimes and photon correlations in an electron gas, and the general conclusion has been that, although correlations substantially reduce the lifetime, ${ }^{3}$ the angular distributions in this simple case are little affected. ${ }^{2}$ Because of its complexity, the problem of interaction effects in real metals has received much less attention. ${ }^{4}$ This is, however, an important question, both in evaluating the experiment as a technique for studying the electronic structure of metals and in obtaining a fundamental understanding of the annihilation process. It is, for instance, of interest to determine whether structure in the angular distribution predicted by the independentparticle model persists in the correlated system or whether the pronounced anisotropy which might be expected in a complex uniaxial metal is significantly

* Work was performed partially in the Ames Laboratory of the U. S. Atomic Energy Commission. Contribution No. 2197.

$\dagger$ Present address: Oak Ridge National Laboratory, Oak Ridge, Tenn.

${ }^{1}$ S. De Benedetti, C. E. Cowan, W. R. Konneker, and H. Primakoff, Phys. Rev. 77, 205 (1950).

${ }^{2}$ J. P. Carbotte and S. Kahana, Phys. Rev. 139, A213 (1965).

3 J. P. Carbotte, Phys. Rev. 155, 197 (1967)

${ }^{4}$ C. K. Majumdar, Phys. Rev. 140, A227 (1965). reduced by the strong interaction between the electron and the positron at the time of annihilation. In order to study these questions experimentally, it is clearly advantageous to use a uniaxial metal with a highly anisotropic electronic structure, and this indicated the choice of yttrium, a hexagonal close-packed transition metal, for an evaluation of the independent-particle theory of positron annihilation. The calculation of the angular distribution of the photons from the independent-particle model was undertaken by Loucks, ${ }^{5}$ and his analysis, as well as preliminary experimental results, ${ }^{6}$ have previously been published.

The experimental determination of detailed information about the electronic structure of the rare-earth metals has proved a formidable problem. The relatively low purity of available samples has, so far, prohibited the use of the conventional techniques of Fermi-surface determination. In addition, the magnetic ordering at low temperatures severely restricts the number of methods which may be used to study the electronic structure in the paramagnetic phase, and it is this which is of primary interest in understanding the relationship between the conduction electrons and the magnetic structures. Fortunately, it has recently proved possible to perform a priori band-structure calculations on transition metals, and these have given results in good agreement with Fermi-surface dimensions derived from experiments. Such calculations have now been carried out for rare-earth metals ${ }^{5,7,8}$ and have greatly clarified

${ }^{5}$ T. L. Loucks, Phys. Rev. 144, 504 (1966).

${ }^{6}$ R. W. Williams, T. L. Loucks, and A. R. Mackintosh, Phys. Rev. Letters 16, 168 (1966).

${ }_{7}^{7}$ J. O. Dimmock and A. J. Freeman, Phys. Rev. Letters 13, 750 (1964).

${ }_{8}^{8}$ A. J. Freeman, J. O. Dimmock, and R. E. Watson, Phys. Rev. Letters 16, 94 (1966). 


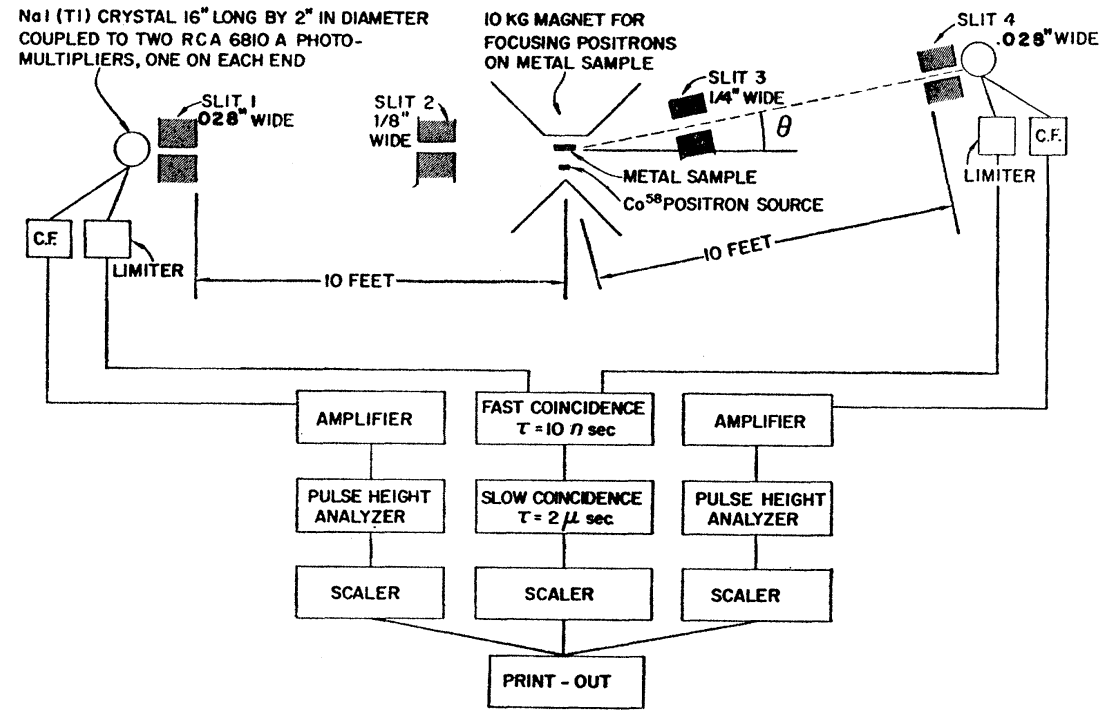

Fig. 1. Schematic diagram of the apparatus.

our understanding of the conduction electrons. The companion paper by Keeton and Loucks ${ }^{9}$ (I) gives the results of a thorough study of the band structure of the heavy rare-earth metals, and the positron-annihilation experiments are an attempt to verify experimentally some of their conclusions while studying those similarities and differences among the electronic structures

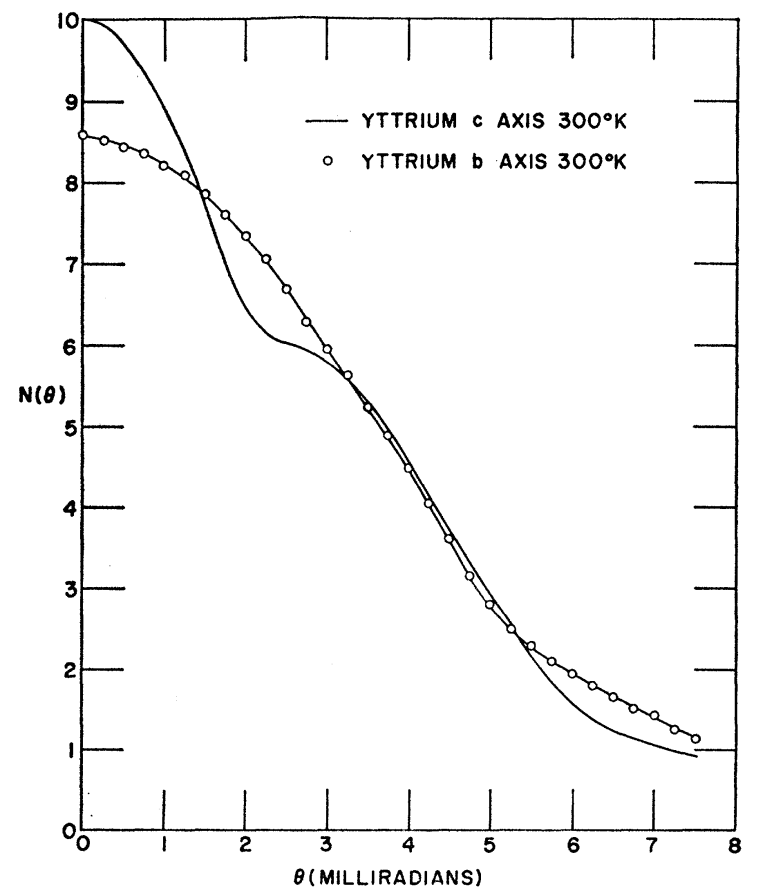

FIG. 2. The angular distribution of photon coincidences from single crystals of $\mathrm{Y}$. The experimental points for the $c$-axis curve are shown in Fig. 7.

${ }^{9}$ S. C. Keeton and T. L. Loucks, preceding paper, Phys. Rev. 168,672 (1968), referred to as I. of these metals which are manifested in their magnetic properties.

The techniques used in these experiments were conventional, but they were developed to a point where a high resolution and statistical accuracy were attained. This experimental procedure and the preparation and characteristics of the samples are briefly described in the next section, followed by a presentation of the results on monocrystalline samples of the metals Y, Gd, $\mathrm{Tb}, \mathrm{Dy}, \mathrm{Ho}$, and Er, and an equiatomic alloy of Ho and Er. The experimental results for $\mathrm{Y}$ are then used, together with the calculations of Loucks, to discuss the applicability and limitations of the independentparticle theory of positron annihilation and to evaluate the experiment as a technique for studying the electronic structure of metals. Finally, those aspects of the electronic structure of the rare earths responsible for the characteristic features of the angular distributions are analyzed and shown to be related to the magnetic properties of these metals.

\section{EXPERIMENTAL PROCEDURE}

The angular correlation of the annihilation photons from the rare-earth crystals was measured with a conventional parallel slit system, shown schematically in Fig. 1. This apparatus has been described in detail by Gustafson. ${ }^{10}$ The monocrystalline samples, in the form of disks approximately $10 \mathrm{~mm}$ in diam were glued to a copper rod, which was mounted in thermal contact with a constant temperature bath in the evacuated tail of a Dewar system. The positron source, approximately $0.1 \mathrm{Ci}$ of $\mathrm{Co}^{58}$ with a half-life of 70 days, was plated on foil and mounted close to the sample but shielded from the detectors by lead slits. Because of the relatively

${ }^{10} \mathrm{D}$. R. Gustafson, Ph.D. thesis, Iowa State University, 1964 (unpublished). 

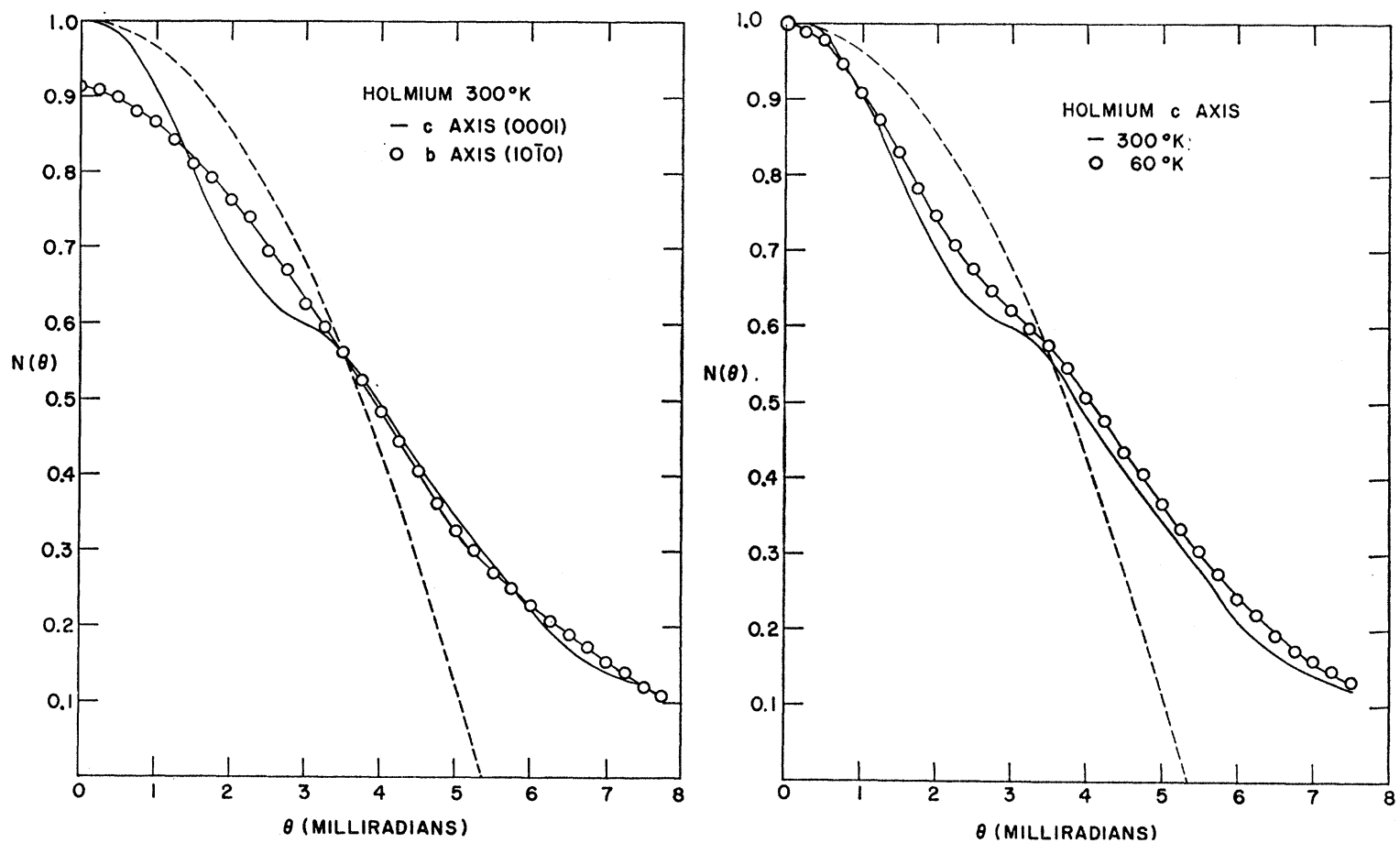

Fig. 3. (a) Photon coincidences as a function of angle in Ho at $300^{\circ} \mathrm{K}$. The dashed curve is the parabola corresponding to three free electrons per atom. (b) The temperature dependence of the coincidence distribution in the $c$-axis crystal.

small size of the samples used, focusing of the positrons with a 10-kG Varian electromagnet, which had a field gradient of approximately $1 \mathrm{kG} / \mathrm{cm}$ in the vicinity of the sample, was necessary to utilize the source to the maximum effect.

The annihilation photons were selected by the slit system shown in Fig. 1. Slits 1 and 2 were fixed while slits 3 and 4 were mounted on a movable arm which was pivoted about an axis through the sample. Slits 2 and 3 shielded the detectors from the $\mathrm{Co}^{58}$ source while slits 1 and 4 defined an angular resolution of $0.23 \mathrm{mrad}$ for the apparatus.

Each detector consisted of two optically coupled $\mathrm{NaI}(\mathrm{Tl})$ scintillation crystals, each $2 \mathrm{in}$. in diam and 8 in. long. They were encapsulated in a thin-walled aluminum cylinder with optically coupled glass windows
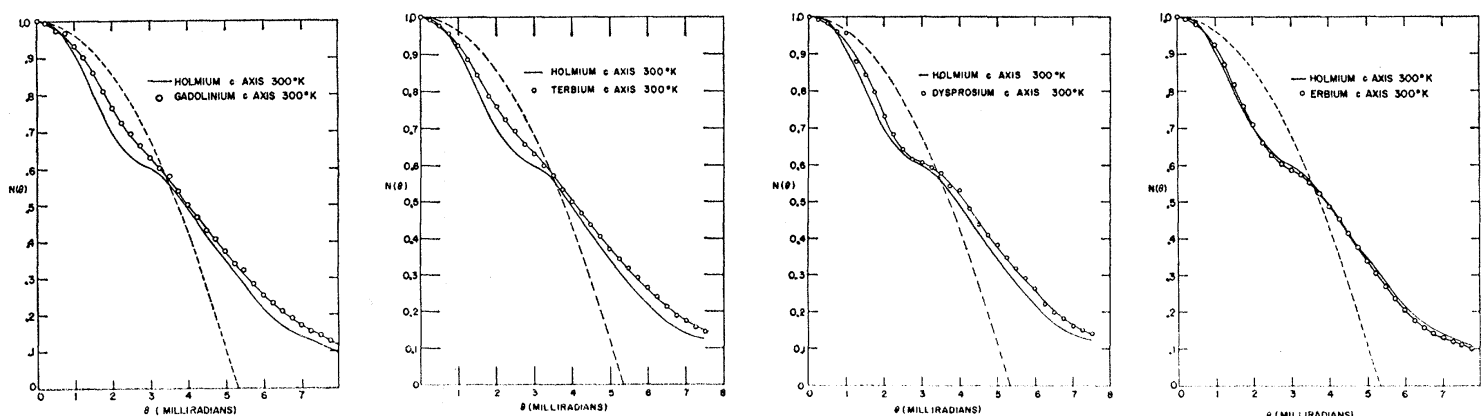

Fig. 4. The angular distribution of photon coincidences from $c$-axis crystals of $\mathrm{Gd}, \mathrm{Tb}, \mathrm{Dy}$, and Er, compared with that in $\mathrm{Ho}$.

${ }^{11}$ H. E. Nigh, Ph.D. thesis, Iowa State University, 1963 (unpublished). 


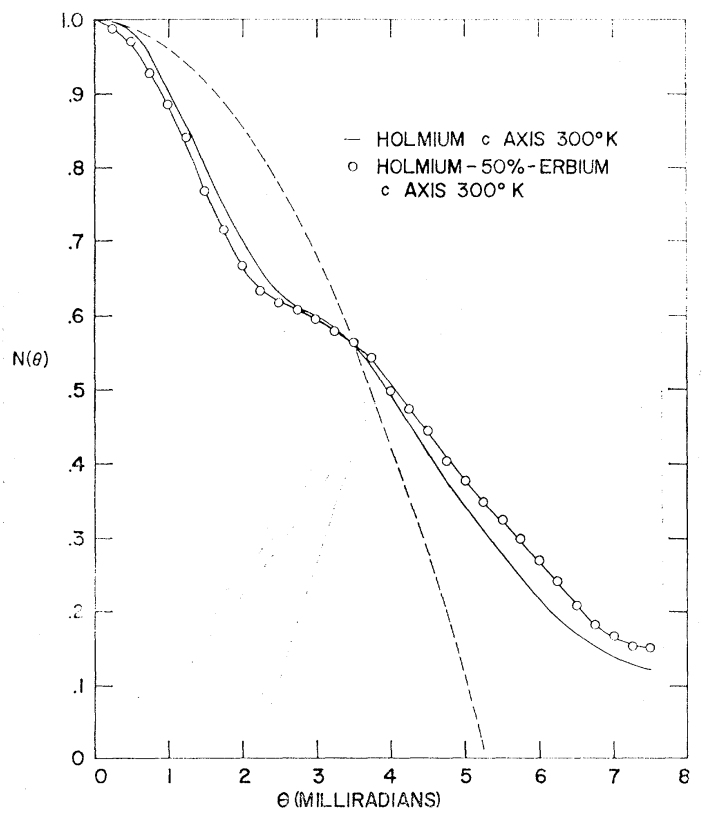

FIG. 5. The angular distribution of photon coincidences from a $c$-axis crystal of an equiatomic alloy of Ho and Er.

suspended and annealed in a high-temperature furnace. In this way, it was possible to produce monocrystalline grains sufficiently large that oriented samples in the form of disks, approximately $10 \mathrm{~mm}$ in diam and $3 \mathrm{~mm}$ thick, could be spark cut from them. These samples were then electropolished in order to produce a smooth, flat, undamaged surface, and kept in vacuio until ready for use.

\section{EXPERIMENTAL RESULTS}

The angular distributions of photon coincidences from monocrystalline disks of $\mathrm{Y}$ with their axes along the $c$ direction (0001) and $b$ direction (1010) are shown in Fig. 2. We have normalized the curves to the same area, corresponding to the fact that, for any orientation, the sum of photon pairs over all angles is equal to the total number of annihilating positrons. The total number of coincidences observed at zero angle in each of the samples is given in Table I. The anisotropy in the electronic structure is clearly manifested in the angular distributions. The $b$-axis curve is quite close to the freeelectron parabola corresponding to three conduction electrons per atom, but the number of coincidences in the $c$ direction drops sharply at low angles, with a hump at about $3 \mathrm{mrad}$.

Very similar results are observed in the paramagnetic phase of Ho, as shown in Fig. 3(a). At $60^{\circ} \mathrm{K}$, however, in the helically ordered magnetic phase, the $c$-axis distribution is somewhat modified in that the drop in coincidences at low angles and the hump are both less pronounced [Fig. 3(b)]. The $c$-axis distributions for the other rare-earth metals studied, Gd, Tb, Dy, and Er, are compared with the Ho results in Fig. 4. The distri- butions all show the same qualitative features and the latter two have a degree of structure comparable with that of $\mathrm{Ho}$ in the paramagnetic phase. Gd and $\mathrm{Tb}$, on the other hand, more closely resemble the spiral phase of Ho.

The $c$-axis results for an equiatomic alloy of Ho and Er are shown in Fig. 5. The distribution is close to those of the constituent metals, but it is noteworthy that the decrease in the count rate at low angles is somewhat faster than is observed in either Ho or Er.

\section{DISCUSSION}

\section{Independent-Particle Model}

In this subsection we shall first consider the extent to which the independent-particle model of positron annihilation is able to account for the experimental results. There is already available a considerable body of evidence on this question..$^{12}$ In particular, Melngailis and De Benedetti ${ }^{13}$ have used the orthogonalizedplane-wave (OPW) method to calculate the angular distributions in $\mathrm{Li}$ and have shown that the results of Donaghy et al..$^{14}$ can be accounted for, provided that the theoretical angular correlation curve is multiplied by
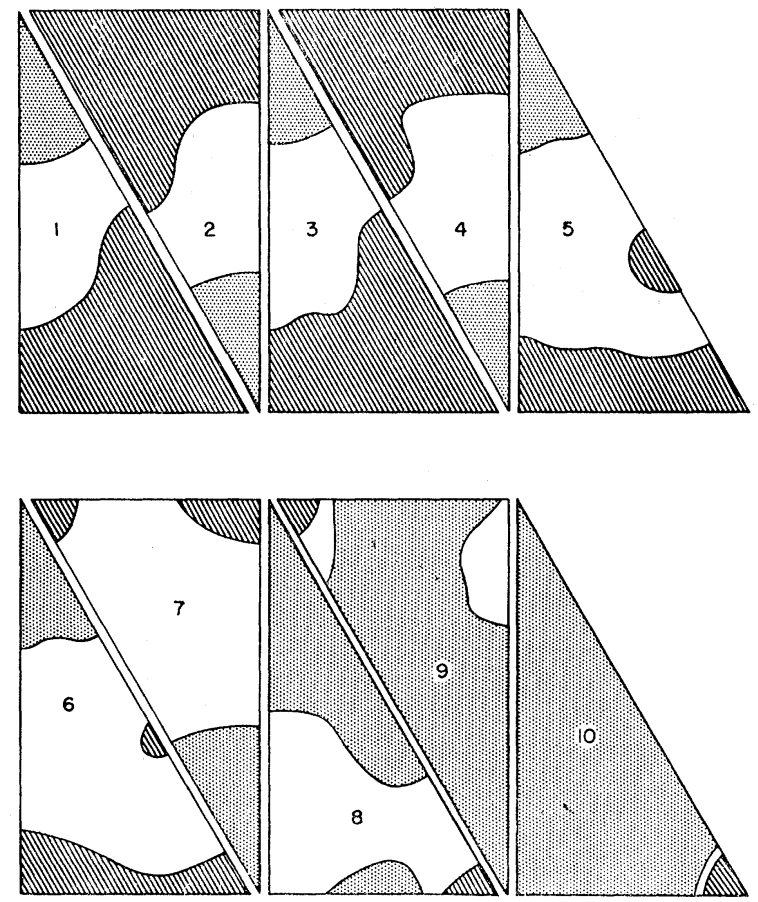

FIG. 6. Fermi surface cross sections for $\mathrm{Y}$ normal to the $c$ direction, after Ref. 5 .

${ }_{12}$ Proceedings of the Positron Annihilation Conference, Detroit, 1965, edited by A. T. Stewart and L. O. Roellig (Academic Press Inc., New York, 1966).

${ }^{13} \mathrm{~J}$. Melngailis and S. De Benedetti, Phys. Rev. 145, 400 (1966).

14 J. J. Donaghy, A. T. Stewart, D. M. Rockmare, and J. H. Kusmiss, in Proceedings of the Ninth International Conference on Low Temperature Physics, Columbus, Ohio, edited by J. G. Daunt et al. (Plenum Press, Inc., New York, 1965). 


\begin{tabular}{|c|c|c|c|}
\hline Sample & $\begin{array}{l}\text { Number of } \\
\text { coincidences }\end{array}$ & Sample & $\begin{array}{l}\text { Number of } \\
\text { coincidences }\end{array}$ \\
\hline$Y .(c$ axis $)$ & 83093 & Gd ( $c$ axis $)$ & 26777 \\
\hline$Y(b$ axis $)$ & 63655 & $\mathrm{~Tb}(c$ axis $)$ & 50825 \\
\hline Ho ( $c$ axis) & 102664 & Dy ( $c$ axis) & 17333 \\
\hline Ho ( $b$ axis) & 61199 & $\operatorname{Er}(c$ axis $)$ & 127759 \\
\hline $\begin{array}{l}\text { Ho ( } c \text { axis }) \\
60^{\circ} \mathrm{K}\end{array}$ & 94162 & $\begin{array}{l}\mathrm{Er}-50 \%-\mathrm{Ho} \\
\quad(c \text { axis })\end{array}$ & 48458 \\
\hline
\end{tabular}

the free-electron enhancement factor of Kahana. ${ }^{2}$ They emphasize that the results are critically dependent on the correct calculation of the wave functions and point out that the use of the free-electron enhancement factor in a real metal requires justification. Furthermore, in a metal with such a small anisotropy the correct subtraction of the core annihilation is of great importance.

A detailed calculation of the angular distribution for $\mathrm{Y}$ in the $c$ direction has been made by Loucks, ${ }^{5}$ who used the augmented-plane-wave (APW) method. In the independent-particle model, the number of coincidences at an angle $\theta$ is proportional to

$$
N(\theta)=N\left(\frac{\hbar p_{z}}{m c}\right)=\int_{-\infty}^{\infty} \int_{p_{x}} d p_{x} d p_{y} \sum_{\mathbf{k}} \Gamma(\mathbf{p}, \mathbf{k}),
$$

where the sum is over all occupied states and

$$
\Gamma(\mathbf{p}, \mathbf{k})=\left|\int \psi_{\mathbf{k}}(\mathbf{r}) \psi_{+}(\mathbf{r}) e^{-i \mathbf{p} \cdot \mathbf{r}} d \mathbf{r}\right|^{2}
$$

The energy eigenvalues for the electrons were calculated

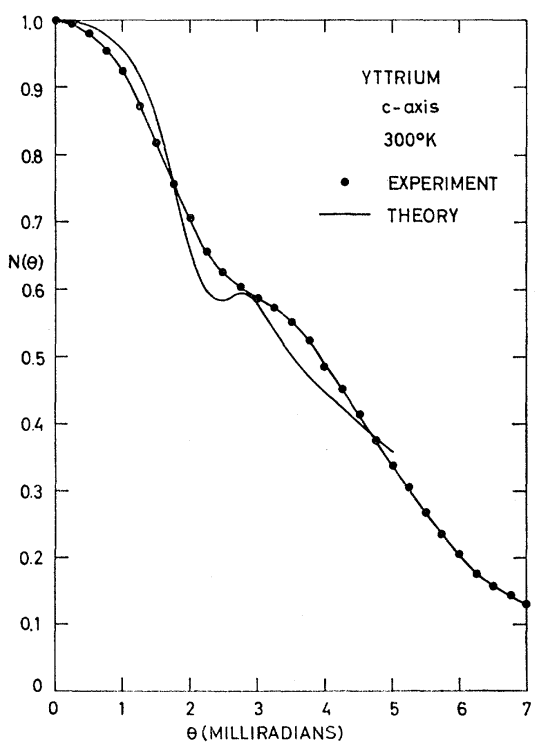

FIG. 7. The calculated and observed coincidence distributions in a $c$-axis $\mathrm{Y}$ crystal. The calculations have been corrected for experimental resolution.

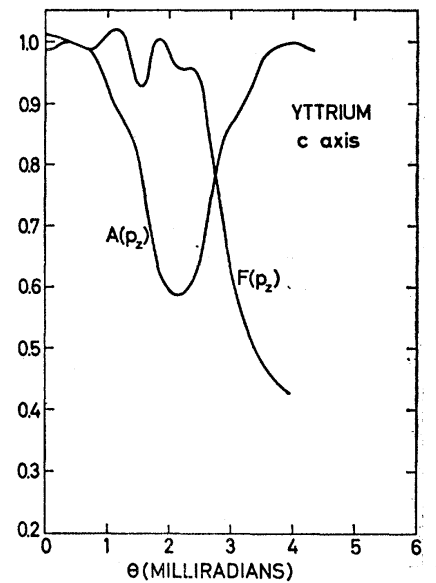

FIG. 8. The functions $A\left(p_{z}\right)$ and $F\left(p_{z}\right)$ for $\mathrm{Y}$, calculated from the results of Loucks (private communication).

using a muffin-tin potential with Slater exchange, and sections of the resulting Fermi surface are shown in Fig. 6. The electron eigenfunctions $\psi_{\mathrm{k}}(\mathbf{r})$ were expanded as a linear combination of APW, and the ground-state positron wave function $\psi_{+}(\mathbf{r})$ was obtained from a numerical solution of the Schrödinger equation using the muffin-tin potential without exchange. Thepublished results of Ref. 5 were obtained with a wave function expansion of $22 \mathrm{APW}$, but more recent calculations have been performed with 32 APW and differ only very slightly from the earlier results. This indicates that the convergence of the calculated energy levels is good and that the wave functions for the assumed potential are accurately determined.

In order to compare the calculated and experimental angular distributions, the former must be corrected for the finite resolution of the apparatus under the conditions of the experiment. The geometrical resolution has already been briefly discussed, but a more important limitation is placed on the resolution by the thermal motion of the positron, which is neglected in the calculations. The positron motion may be represented by a distribution function of the form $\exp \left(-\hbar^{2} k_{+}{ }^{2} / 2 m k T\right)$, so that the resolution function for the system is well approximated by

$$
P(\theta)=\frac{1}{\sigma \sqrt{ } \pi} \exp \left(-\theta^{2} / \sigma^{2}\right),
$$

where $\sigma$ includes both the effects of the positron motion and the, much smaller, geometrical resolution. At $300^{\circ} \mathrm{K}, \sigma$ has the value 0.35 milliradians if $m$ is taken as the free positron mass. The experimental results are compared with the calculated distribution, convoluted with this resolution, in Fig. 7.

Since the main features of the experimental results are qualitatively predicted by theory, it is of interest to determine what aspects of the electronic structure cause the rather unusual structure in the distributions. The conduction-electron wave functions are Bloch functions 


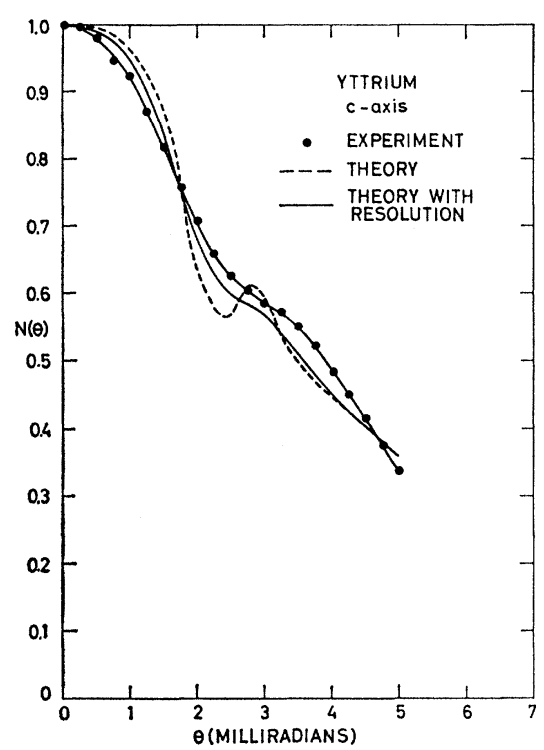

FIG. 9. The experimental photon distribution for the $c$-axis $Y$ crystal, compared with the theoretical distribution for perfect resolution, and for a resolution corresponding to $\sigma=0.55$ mrad.

and, neglecting the thermal motion of the positrons, $\psi_{+}(\mathbf{r})$ has the periodicity of the lattice. We may therefore write Eq. (2) as

$$
\begin{aligned}
\Gamma(\mathbf{p}, \mathbf{k})=\left|\int \psi_{+}(\mathbf{r}) u_{\mathbf{k}}(\mathbf{r}) e^{i(\mathbf{k}-\mathbf{p}) \cdot \mathrm{r}} d \mathbf{r}\right|^{2} \\
=B_{\mathbf{g}}(\mathbf{p}, \mathbf{k}) \delta(\mathbf{p}-\mathbf{k}-\mathbf{g}),
\end{aligned}
$$

which implies that the photon pair produced by the annihilation of an electron of wave vector $\mathbf{k}$ must have a momentum in the set of values $\hbar(\mathbf{k}+\mathbf{g})$, where $\mathbf{g}$ is any reciprocal lattice vector. Equation (1) can therefore be written formally

$$
N\left(p_{z}\right)=A\left(p_{z}\right) F\left(p_{z}\right),
$$

where $A\left(p_{z}\right)$ is the number of distinct occupied states in a plane section through the periodic zone scheme at a perpendicular distance $p_{z}$ from the origin in $\mathbf{k}$ space. $A\left(p_{z}\right)$ therefore necessarily has the periodicity of the reciprocal lattice. $F\left(p_{z}\right)$ depends upon the electron and positron wave functions and, in the hypothetical case where the Bloch functions are plane waves with wave vectors in the primitive Brillouin zone while $\psi_{+}(\mathbf{r})$ is constant, it is constant in the primitive zone and zero outside. $A\left(p_{z}\right)$ for the $c$ direction in $\mathrm{Y}$ may readily be deduced from the Fermi surface sections of Fig. 6 and the result is shown in Fig. 8. From the calculated $N\left(p_{z}\right), F\left(p_{z}\right)$ may then be deduced and is also shown in Fig. 8. It is clear from these results that the rapid fall in $N\left(p_{z}\right)$ at low angles is due to the rapid decrease in the number of occupied states with $k_{z}$, which may be observed in Fig. 6. The same feature, together with the precipitous fall of $F\left(p_{z}\right)$ beyond the primitive Brillouin zone boundary, accounts for the hump in the photon distribution. Both of the characteristic features of the angular correlation measurements in the $c$ direction are therefore manifestations of the same feature of the electronic structure. The rapid drop in $F\left(p_{z}\right)$ beyond the primitive zone boundary probably reflects the rapid decrease of the amplitude of the positron wave function near the nucleus, ${ }^{5}$ due to the Coulomb repulsion, which has the effect of strongly attenuating terms with $\mathbf{g}$ different from zero in Eq. (3).

We conclude, therefore, that the independentparticle model provides a good first approximation to the theory of electron-positron annihilation in $\mathrm{Y}$ and that the characteristic features of the photon distribution reflect the rapid change in the occupation of states with $k_{z}$, which is a consequence of the rather flat electron and hole sheets normal to the $c$ axis. It remains to explain, however, why the structure in the theoretical distribution is considerably suppressed in the experimental results. Although previous experience ${ }^{15}$ has shown that APW calculations of Fermi-surface dimensions generally agree well with those determined from experiment, it is possible that the matrix elements in (2) are incorrect because of the inadequacy of the wave functions. The fact that the calculations with 22 and 32 APW agree very closely would seem to militate against this possibility, however, since it implies that the calculated wave functions are a very good approximation to the independent-particle wave functions in the assumed potential. An important ingredient which has been omitted from the calculations is the Coulomb correlation in the electron-positron system, and it seems more likely that the discrepancy between theory and experiment due to this. In Fig. 9 we have plotted the experimental results with the calculated distributions for perfect resolution, and for $\sigma=0.55$ milliradians. The latter, which is considerably greater than the true experimental resolution, was found to give a distribution which most closely resembles the experimental results, and the degree of structure is very similar to that observed in practice, although the agreement is not exact. Such a degradation of the resolution could be explained by a positron effective mass of approximately $2.5 \mathrm{~m}$. However, there seem to be no grounds for expecting such a large increase in the mass, ${ }^{16}$ even though rather large effective masses have apparently been observed in the alkali metals, ${ }^{17}$ and it seems more likely that the suppression of the structure is due to a decrease in the anisotropy of the conduction-electron gas in the vicinity of the positron. This implies that the Coulomb correlation imposes a fundamental limitation on the resolution of the experiment, apart from that due to possible nonthermalization of the positrons, ${ }^{18}$ although the approxi-

${ }^{15}$ T. L. Loucks, A ugmented Plane Wave Method (W. A. Benjamin, Inc., New York, 1967).

${ }_{16}$ D. R. Hamann, Phys. Rev. 146, 277 (1966).

${ }^{17}$ A. T. Stewart, J. B. Shand, and S. M. Kim, Proc. Phys. Soc. (London) 88, 1001 (1966).

${ }_{18}$ S. M. Kim, A. T. Stewart and J. P. Carbotte, Phys. Rev. Letters 18, 385 (1967). 
mation of the complexities of this effect by a constant degradation of the experimental resolution is clearly a great oversimplification. If this is correct, it is an important consideration in evaluating positron annihilation as a technique for studying the electronic structure of metals. Experiments over a wide temperature range would clearly assist in further clarifying this question.

\section{Electronic Structure of the Rare-Earth Metals}

Band-structure calculations $s^{5,9}$ indicate that there is a strong similarity between the electronic structure of $\mathrm{Y}$ and that of the heavy hcp rare-earth metals, and it is not therefore surprising that the angular distributions are also similar. The rapid fall in the number of coincidences at small angles and the hump in the $c$-axis results again reflect the rather flat electron and hole sheets of the Fermi surface, and the differences in these features between the different metals can be correlated with the band structures and magnetic properties.

The photon distributions from positron annihilation have not been calculated for the magnetic rare-earth metals, but $A\left(p_{z}\right)$ can readily be deduced from the Fermi surfaces presented in I. The results for Gd and Er are compared with $\mathrm{Y}$ in Fig. 10 and, as may be seen from Figs. 5(b) and 5(d) of I, Dy is very similar to Er. The similarities between the photon distributions for these metals can then immediately be explained, provided that the reasonable assumption is made that the conduction-electron wave functions do not differ greatly among them. We may also conclude that Ho has a very similar Fermi surface. On the other hand, Gd is somewhat different, and the relatively slow decrease in $A\left(p_{z}\right)$ explains why the coincidence rate falls more slowly at low angles. This behavior is in turn a consequence of the fact that the second lowest doubly degenerate level at $L$ is above the Fermi level in most of the metals (see Fig. 6 of I) but below it in Gd so that the number of occupied states near the hexagonal face of the primitive zone is relatively high.

This feature also strongly influences the magnetic structures of the hcp rare-earth metals. The formation of a periodic magnetic structure requires that there be a maximum in $J(\mathbf{q})$, the Fourier transform of the indirect exchange coupling, at some nonzero value of $\mathbf{q}$. Such a maximum in $J(\mathbf{q})$ in the $c$ direction has been observed explicitly through measurements of magnon dispersion relations by inelastic neutron scattering. ${ }^{19}$ $J(\mathbf{q})$ is given by an expression of the form

$J(\mathbf{q})=\sum_{n, n^{\prime}, \mathbf{k}} \frac{I_{n n^{\prime}}(\mathbf{k}, \mathbf{k}+\mathbf{q})\left\{f\left[\epsilon_{n}(\mathbf{k})\right]-f\left[\epsilon_{n^{\prime}}(\mathbf{k}+\mathbf{q})\right]\right\}}{\epsilon_{n^{\prime}}(\mathbf{k}+\mathbf{q})-\epsilon_{n}(\mathbf{k})}$,

where $\epsilon_{n}(\mathbf{k})$ is the energy of a Bloch state of wave vector $\mathbf{k}$ in band $n$ in the periodic zone scheme, $I_{n n^{\prime}}(\mathbf{k}, \mathbf{k}+\mathbf{q})$ is a slowly varying function, and $f(\epsilon)$ is the Fermi-Dirac

${ }^{19} \mathrm{H}$. Bjerrum M $\phi l l e r$, J. C. G. Houmann, and A. R. Mackintosh, Phys. Rev. Letters 19, 312 (1967); and (to be published).

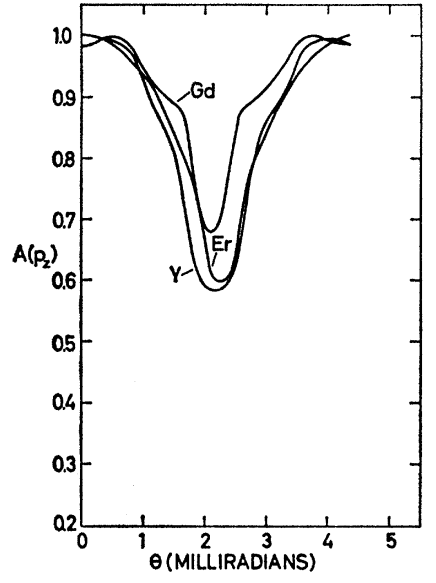

Fig. 10. The function $A\left(p_{z}\right)$ for Gd, Er, and Y calculated from the Fermi-surface sections of Refs. 5 and 9.

distribution. The maximum in $J(\mathbf{q})$ is believed to be due to transitions between states on approximately parallel sheets of the Fermi surface. ${ }^{9}$ The lowering of the level at $L$ below $\epsilon_{F}$ in Gd distorts the Fermi surface in such a way that this feature is eliminated. $J(\mathbf{q})$ in $\mathrm{Gd}$ therefore has a maximum at $\mathbf{q}=0$, and it forms only a ferromagnetic structure. Preliminary band-structure calculations on $\mathrm{Tb}^{19}$ have shown that the second level at $L$ is very close to the Fermi level, and this is consistent with the small hump in the photon distribution and with the fact that $\mathrm{Tb}$ shows only a very weak tendency towards spiral ordering, with an anomalously small $Q$.

The exchange coupling in the spiral phase strongly modifies the electron states in the vicinity of the Fermi surface. This is manifested, for instance, in the transport properties ${ }^{20}$ as it is in the analogous case of $\mathrm{Cr}$ and its alloys. ${ }^{21}$ It also has a considerable effect on the occupancy of the states in $\mathbf{k}$ space, which in turn is reflected in the photon distribution from Ho, as shown in Fig. 6(b). In particular, the annihilation of the flat surfaces normal to the $c$ axis reduces the rapid fall at low angles and the hump, as expected.

The equiatomic alloy of Ho and Er shows a pronounced hump in the angular distribution in the $c$ direction, and correspondingly this alloy forms a periodic magnetic structure down to $4.2^{\circ} \mathrm{K} .{ }^{22}$ The distribution is very little different from those of the constituent metals, and this provides evidence that the concept of a band structure has some validity in such a highly disordered structure. On the other hand, the distribution does not lie between those for the constituent elements so that the change in the potential on alloying apparently causes relative changes in the energy levels and a rigid-band model is not strictly applicable.

${ }^{20}$ A. R. Mackintoch and L. E. Spanel, Solid State Commun. 2, 383 (1964).

${ }^{21}$ A. L. Trego and A. R. Mackintosh, Phys. Rev. (to be published).

${ }^{22}$ G. Shirane and S. J. Pickart, J. Appl. Phys. 37, 1032 (1966). 


\section{CONCLUSIONS}

In this study, we have attempted to determine the extent to which the independent-particle model describes the angular correlation of the photons emitted when positrons and electrons annihilate in metals and, hence, to evaluate the experiment as a technique for studying the electronic structure of metals. We have concluded that the independent-particle model provides a good first approximation to the angular distribution, even in very anisotropic metals, but that some of the detailed structure which the model predicts is probably reduced by the Coulomb correlations in the system.

The electronic structures of the heavy rare-earth metals have been shown to be rather similar, and the angular distributions which we have observed are qualitatively in agreement with the relativistic augmented-plane-wave calculations of Keeton and Loucks. The structure in the $c$-axis distributions has been shown to be related to those aspects of the Fermi surface which are believed to be important in determining the magnetic structures. In particular, the difference between Gd and the other magnetic hcp rare-earth metals can be qualitatively understood. The modification of the
Fermi surface in the helically ordered magnetic phase of Ho has the expected effect of reducing the structure in the $c$-axis angular distribution. The electronic structure of the equiatomic Ho-Er alloy has been shown to be similar to those of the constituent metals although a rigid-band model is not apparently strictly applicable.

\section{ACKNOWLEDGMENTS}

We have been greatly assisted in this work by the interest and encouragement of Professor T. L. Loucks, whose calculations have allowed a much more detailed interpretation of our results than would otherwise have been possible. O. Dietrich kindly performed the convolution of the theoretical distribution with the resolution function. Valuable discussions with Dr. D. R. Gustafson, who constructed much of the apparatus used in this experiment, and the advice and encouragement of Professor D. J. Zaffarano are gratefully acknowledged. The rare-earth metals and alloys used in this work were kindly supplied by B. J. Beaudry and Professor F. H. Spedding, and invaluable help in growing the single crystals was given by Professor S. Legvold and his group.

\title{
Dynamics of Electrons in Solids in External Fields
}

\author{
J. ZAK* \\ Deparlment of Physics and Francis Bitter National Magnet Laboratory, $\uparrow$ Massachuselts \\ Institute of Technology, Cambridge, Massachusetts
}

(Received 11 December 1967)

\begin{abstract}
A quantum-mechanical representation is defined by means of finite translations in direct and reciprocal space. The eigenfunctions of the finite translations are found and their connection with Bloch functions and Wannier functions is established. The new representation is used for describing the motion of a Bloch electron in a magnetic and in an electric field. For the first problem, well-known results are reproduced in an extremely simple and natural way. It is also shown that the representation introduced in this paper gives a straightforward way to compare classical and quantum dynamics for a Bloch electron in external magnetic and electric fields.
\end{abstract}

\section{INTRODUCTION}

$T^{1}$ HE motion of electrons in solids (Bloch electrons) in external magnetic and electric fields has attracted great attention both experimentally and theoretically during all periods in the development of solidstate physics. The reason for this is that very many properties of solids, and in particular their energy spectrum, can be measured by performing experiments in external fields. For example, all the vast field of magneto- and electro-optics and Fermi-surface measurements by the de Haas-van Alphen technique is based on this kind of experiment. It is therefore natural that

* Permanent address : Department of Physics, Technion-Israel Institute of Technology, Haifa, Israel.

$\dagger$ Supported by the U. S. Air Force of Scientific Research. many papers have been published on the motion of Bloch electrons in external fields. ${ }^{1-4}$ The final results derived in the mentioned papers have a very simple physical interpretation and are closely related to the dynamics of unperturbed Bloch electrons. This is a common feature of all theories for the given problem, and physically one should expect that the perturbed motion will in one way or another be connected to the unperturbed one. The mentioned papers ${ }^{1-4}$ differ, however, by their approach to the problem, namely, different

\footnotetext{
${ }^{1}$ W. Kohn, Phys. Rev. 115, 1460 (1959); see this paper for other references.

${ }^{2}$ Laura M. Roth, J. Phys. Chem. Solids 23, 433 (1962).

${ }^{3}$ Gregory H. Wannier, Rev. Mod. Phys. 34, 645 (1962); see this paper for other references.

${ }^{4}$ E. I. Blount, Phys. Rev. 126, 1636 (1962).
} 